\title{
VARIATION OF WITHIN-STEM BIOMETRICAL AND PHYSICAL PROPERTY INDICES OF WOOD FROM CUPRESSUS SEMPERVIRENS L.
}

\author{
Seyyed Khalil Hosseini Hashemi ${ }^{a, *}$ and Behzad Kord ${ }^{b}$
}

\begin{abstract}
Variations in average tracheid dimensions (such as length, diameter, lumen diameter, and wall thickness) and its biometrical ratios including slenderness ratio, flexibility ratio, and Runkell ratio, oven-dry and basic density, longitudinal, radial, tangential, and volume shrinkage, maximum moisture content, and porosity of cypress trees wood (Cupressus sempervirens L. var. horizontalis), which was cultivated in the north of Iran, were studied from the pith to bark (radial position), and along the stem from the base upwards. To measure the mentioned traits, the test specimens were prepared from three stands and 9 discs at different height levels $(5,25$, and $50 \%$ of the total tree height) based on ASTMD143 standard. Results indicated that the tracheid length, tracheid crosssectional dimensions, and its biometrical ratios irregularly varied at each height level, along the stem from the bottom to top, but within the discs, at the same height level biometrical traits in the radial position regularly increased from the pith to bark. Within the tree, wood oven-dry and basic density, longitudinal, radial, tangential, and volume shrinkage varied at each height level, decreasing along the stem from the base upwards. Within the discs, at the same height level, wood density and shrinkage, except for longitudinal shrinkage in the radial position, increased from the pith to bark. The maximum moisture content and porosity varied at each height level, increasing along the stem from the bottom to the top of the tree. Moreover, within the discs, at the same height level in the radial direction porosity decreased and maximum moisture content increased except for at $5 \%$ of height level from the pith to bark.
\end{abstract}

Keywords: Cupressus sempervirens L.; Tracheid dimensions; Biometrical ratios; Wood density; Wood shrinkage; Maximum moisture content; Porosity; Variation within-tree

Contact information; a: Agriculture Research Center, Islamic Azad University, Karaj Branch, P. O. Box: 331485-313, Karaj, Iran; b: Assistant Professor, Department of Wood Science and Paper Technology, Islamic Azad University, Chalous Branch, P. O. Box: 46615-397, Mazandaran, Iran.

*Corresponding author: hashemi@kiau.ac.ir

\section{INTRODUCTION}

Cypress is grown in plantations on sites that are unsuitable for agricultural cultivation, where it produces wood of very good quality (Uzielli and Berti 1979; Tischler 1981; Papamichael and Paraskevopoulou 1982), which is used extensively in furniture, joinery, vine props, shipbuilding, and in building construction, where it is used chiefly as roofing poles (Paraskevopoulou 1991). In Iran, Mediterranean cypress (Cupressus sempervirens L.) occurs in natural forests in the Roodbar, Manjil, Dilaman, Zarringol, Aliabade Katool, Arasbaran, and mainly in Polzoghal (Hassan-Abad in 
Chalous) regions, where it is a very important element of the vegetation, but there is a prohibition against cutting it due to the shortage of this species. Thus plantation trees of cypress (C. sempervirens L.) are as an important substitute resource for the targeted applications, and the trees are cultivated extensively in our country, especially in all of the northern areas, including the Ramsar region.

The wood properties have been reported to depend on such factors as climate, provenance, ecological conditions, as well as wood positions in different parts of tree, between, and within species (Koch 1985).

Wood properties such as density and fiber length determine the end-product quality in industrial processes and are both positively correlated with tear strength (Fuglem et al. 2003).

Paraskevopoulou (1991) studied the variation of wood structure and properties of C. sempervirens var. horizontalis in a natural population in Greece. He indicated that the average specific gravity, over all trees (individual tree values ranged from 0.396 to 0.594 $\mathrm{g} / \mathrm{cm}^{3}$ ), decreased and tracheid length (with individual tree values ranging from 2.32 to $3.00 \mathrm{~mm}$ ) increased across discs from the pith outwards, although the tracheid length decreased from the base of the tree up to $35 \%$ of total tree height, after which it decreased.

Wood density is one of the most important properties (Brazier and Howell 1979), and a commonly or general used indicator of wood quality and cell size, since it is a good predictor of timber strength, stiffness, ease of drying, machining, hardness and related to tracheid properties, pulp yield, and various paper making properties (Elliot 1970; Panshin and De Zeeuw 1980; Jyske et al. 2008). It is affected by the proportion of cell wall material in the wood (cell wall thickness), the cell diameter, and the chemical content of the wood, and is hence dependent on the ratio of cell wall thickness and cell diameter (Cave and Walker 1994; Kibblewhite 1999).

Basic density is closely related to end-use quality parameters such as pulp yield and structural timber strength (Harvald and Olesen 1987). Indeed in many conifers, the basic density of the latewood zone is more than twice that of earlywood; thus, any increase in the proportion of latewood inevitably leads to an increase in whole ring basic density (Elliot 1970; Ward 1975). In a study carried out by Harvald and Olesen (1987) on the variation of basic density within the juvenile wood of Sitka spruce, it was found that basic density decreased with increasing height in the stem. Previous studies also have examined genetic variation among trees for basic density and fiber length in trembling aspen (Populus tremuloides) (e.g., Brown 1961; Einspher et al. 1967; Yanchuk et al. 1984).

It has been found that pith-to-bark variation in longitudinal shrinkage is a key factor for distortion in timber drying. However, the difference in the longitudinal shrinkage between two faces of the timber can explain spring or bow characteristics much better when the variation in shrinkage along the timber is considered (Kilger et al. 2003). Deresse et al. (2002) found that longitudinal shrinkage of red pine (Pinus resinosa Ait.) declined significantly with increasing growth ring number from the pith, and the influence of the growth ring number changed significantly at around 15th growth ring, with the longitudinal shrinkage leveling off after that. Also Herritsch (2007) showed 
similar trends for radiata pine, where tangential shrinkage remained relatively constant in the transition wood and the outerwood.

The variation in anisotropic shrinkage of plantation-grown Pinus radiata wood was studied by Wang et al. (2008). They showed that longitudinal shrinkage varied from 0.02 to $2.34 \%$, with peak values near the pith, decreasing towards the bark, and with more pronounced variation at $0.1 \mathrm{~m}$ height. Also the longitudinal shrinkage showed a decreasing trend with increasing height in the stem above the ground. Tangential and radial shrinkage were found to increase with growth ring number from the pith, but the variation along the stem height did not show a clear trend.

As is well known, moisture content (MC) of sapwood is significantly higher than that of heartwood in coniferous trees. The MC is important because of its direct relation to the weight and processing of the timber and due to the fact that it varies considerably among species (Haygreen and Bowyer 1982). The green density of Cupressus luistanica wood is $0.91 \mathrm{~g} / \mathrm{cm}^{3}$ with a moisture content of $155 \%$ for freshly felled trees. The moisture content is up to $105 \%$ in the heartwood, whereas sapwood moisture content averaged $180 \%$ but with some samples as high as $225 \%$ (Bannister and Orman 1960).

There is increasing demand for new species from fast-growth plantations as alternative timbers for coniferous wood, but there is little or no information about the variation of wood tracheid dimensions and its biometrical ratios, density, shrinkage, and above mentioned properties within stem and among trees in cypress. These characteristics are a means of assessing suitability for pulp and paper making and the dimensional stability of wood. Thus, the aim of the present study was to investigate the variation of physical and biometrical properties within tree stem of wood from plantation Mediterranean cypress (C. sempervirens L.) species growing under fast-growth conditions in Iran.

\section{EXPERIMENTAL}

\section{Materials}

All the cypress trees (C. sempervirens L.) were randomly selected, taking into account stem straightness and the absence of obvious decay, and cypress wood was selected according to TS 2476, as defect-free, clear, and normally grown (without zone lines, reaction wood, decay, and insect damage, or fungal infection) wood from a plantation in the northern city of Ramsar, Iran. It was grown on a Mediterranean climate at the elevation of 200-250 meters above see level with geographical direction of $36^{\circ} 56^{\prime}$ - $37^{\circ} 49^{\prime} \mathrm{N}$ and $50^{\circ} 27^{\prime}-50^{\circ} 43^{\prime} \mathrm{E}$. Three trees were cut from one provenience. The pattern clay of the study area of cypress is lime and dolomite. The mean annual precipitation and temperature of this area is usually about $1200 \mathrm{~mm}$ and $18{ }^{\circ} \mathrm{C}$.

The characteristics of the study C. sempervirens L. trees are listed in Table 1.

Table 1. Characteristics of the Study C. sempervirens L. Trees

\begin{tabular}{|c|c|c|}
\hline Tree No. & Diameter $(\mathrm{cm})$ & Height $(\mathrm{m})$ \\
\hline 1 & 24.52 & 11 \\
\hline 2 & 22.29 & 12 \\
\hline 3 & 22.29 & 15 \\
\hline
\end{tabular}




\section{Preparation of Test Samples}

In each tree, sample discs of $30 \mathrm{~cm}$ length were taken from three height levels, at $5 \%, 25 \%$, and $50 \%$ of the total tree height. Rough boards at $25 \mathrm{~mm}$ thickness were radially sawn in the four direction of radius. Then test specimens were cut from these rough boards (Fig. 1) having dimensions of $20 \times 20 \times 20 \mathrm{~mm}$ according to ASTM D14394 and used for measuring the oven-dry and basic density, longitudinal, radial, tangential, and volume shrinkage, maximum moisture content, and porosity.

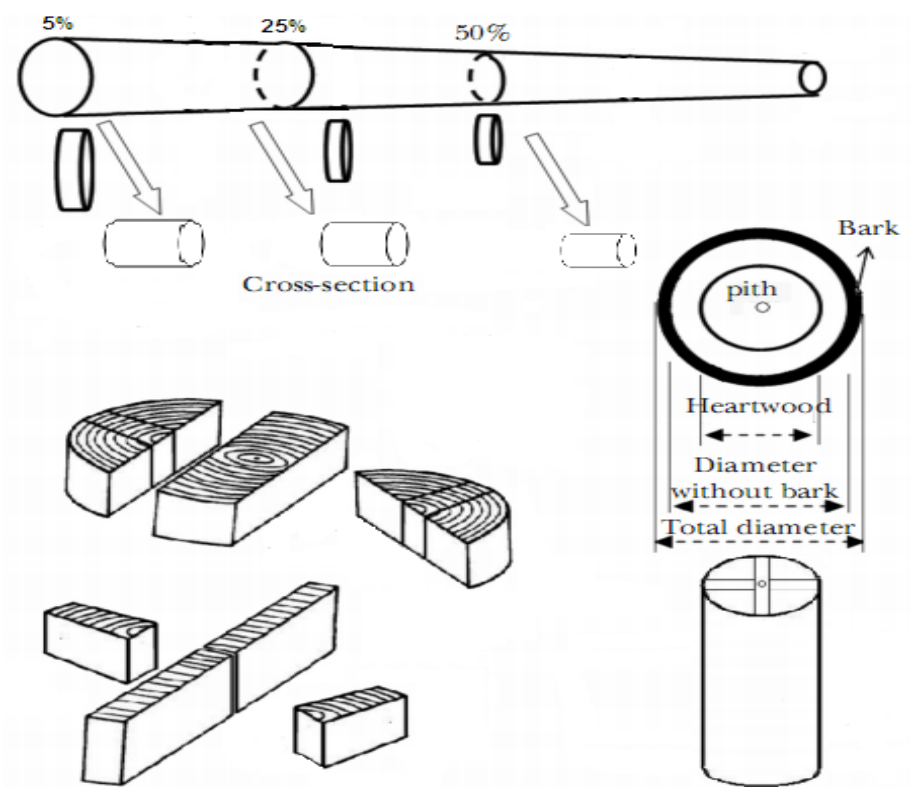

Figure 1. Specimen preparation from the discs

Specimens were randomly used in our experiments. From each sample disc in each tree, 5 specimens in $5 \%$ of height level, 4 specimens in $25 \%$ of height level, and 3 specimens in $50 \%$ of height level were collected randomly, depending on the tree diameter at the three height levels of the stem. In sum, $12(5+4+3)$ cubic pieces $(20 \times$ $20 \times 20 \mathrm{~mm}$ ) were selected from each tree, and three discs randomly from the pith to the bark and finally were tested.

The specimens were soaked in distilled water for $72 \mathrm{~h}$ to ensure that their moisture content was above the fiber saturation point. Then the dimensions in all three principal directions were measured with a digital caliper to the nearest $0.001 \mathrm{~mm}$. Specimens were weighed to the nearest $0.001 \mathrm{~g}$ for saturated weight, and the saturated volume was calculated based on these dimension measurements. Finally, the samples were oven dried at $103 \pm 2{ }^{\circ} \mathrm{C}$ to $0 \%$ moisture content. After cooling in desiccators, the oven-dry weights of the specimens were measured. The values of the wood oven-dry and basic density, maximum moisture content, porosity and longitudinal, radial, tangential, and volume shrinkage in percentage were calculated using the following equations,

$$
\operatorname{MC}(\max )=\left[\left(M_{S}-M_{O}\right) \div M_{O}\right] \times 100
$$

where $M C$ (max) is the maximum moisture content, $M_{O}$ is the oven dried weight, and $M_{S}$ is the saturated weight of specimen. 


$$
D_{O}=\left(M_{O} \div V_{O}\right) \times 100
$$

where $D_{O}, M_{O}$, and $V_{O}$ are the oven dried density, weight and volume of specimen respectively,

$$
D_{B}=\left(M_{O} \div V_{S}\right) \times 100
$$

where $D_{B}$ is the basic density, $M_{O}$ is the oven dried weight and $V_{S}$ is the saturated volume of specimen,

$$
C=\left(1-0.67 D_{O}\right) \times 100
$$

where $C$ is the porosity, $D_{O}$ is the oven-dry density, and

$$
\begin{aligned}
& B_{L}=\left[\left(L_{S}-L_{O}\right) / L_{S}\right] \times 100 \\
& B_{R}=\left[\left(R_{S}-R_{O}\right) / R_{S}\right] \times 100 \\
& B_{T}=\left[\left(T_{S}-T_{O}\right) / T_{S}\right] \times 100 \\
& B_{V}=\left[\left(V_{S}-V_{O}\right) / V_{S}\right] \times 100
\end{aligned}
$$

where $B_{L}, B_{R}, B_{T}$, and $B_{V}$ are the longitudinal, radial, tangential, and volume shrinkage, $L_{O}, R_{O}, T_{O}$, and $V_{O}$ are the oven dried dimensions, and $L_{S}, R_{S}, T_{S}$, and $V_{S}$ are the saturated dimensions.

From each sample disc in each tree, 10 specimens $(2 \times 2 \times 10 \mathrm{~mm})$ in $5 \%$ of height level, 10 specimens in $25 \%$ of height level, and 10 specimens in $50 \%$ of height level were collected randomly, depending on the tree diameter at the three height levels of the stem, where distance of between selected samples decreased with increasing height in the stem above the ground. In sum, $30(10+10+10)$ splinter pieces $(2 \times 2 \times 10 \mathrm{~mm})$ were selected from each tree, and three discs were taken randomly from the pith to the bark and finally were tested.

Samples for tracheid dimensions measurements were macerated in a mixture (1:1) of $30 \%$ of hydrogen peroxide and glacial acetic acid in a $64{ }^{\circ} \mathrm{C}$ oven for 24 hours (Franklin 1964 modified method in order to lessen the reduction in tracheid length).

After maceration samples were washed with distilled water, and the $2 \times 2 \times 10$ $\mathrm{mm}$ splinters were shaken gently in the distilled water until the individual tracheids of the wood were separated. From each splinter 2 slides were prepared and 10 whole tracheids on each slide were measured. Each slide was projected on an Olympus research microscope using a $1 \times$ eyepiece and $4 \times$ objective lens for tracheid length and at a $10 \times$ objective lens for cross-sectional dimension. First the image of each tracheid was calibrated on monitor board with screw micrometer, and tracheid dimensions were determined with special plastic slide rule. The special transfer ratio was determined on the basis of microscope magnification and monitor magnification with a special microscope slide and on the monitor board. Then, the mean of tracheid length raw data $(\mathrm{mm})$ was multiplied with a 5.3844153/1000 transfer ratio, and the mean of crosssectional dimension raw data $(\mu \mathrm{m})$ was multiplied with a 2.1428571 transfer ratio. 
The biometrical ratios such as slenderness ratio $\left(S_{R}\right)$, flexibility ratio $\left(F_{R}\right)$, and Runkel ratio $\left(R_{R}\right)$ of tracheids were calculated as follows (Ogbonnaya et al. 1997),

$$
\begin{aligned}
S_{R} & =\frac{T_{L}}{T_{D}} \\
F_{R} & =\frac{T_{L D}}{T_{D}} \times 100 \\
R_{R} & =\frac{2 T_{W T}}{T_{L D}} \times 100
\end{aligned}
$$

where $T_{L}, T_{D}, T_{L D}$, and $2 T_{W T}$ are the tracheid length, tracheid diameter, tracheid lumen diameter, and tracheid two-wall thickness, respectively.

\section{Statistical Analysis}

Statistical analysis was conducted using the SPSS program in conjunction with analysis of variance (ANOVA). Duncan's multiple range test (DMRT) was used to test statistical significance at the $\alpha=0.05$ level.

\section{RESULTS AND DISCUSSION}

\section{Within-Tree Variation}

The results of ANOVA indicated that radial position at $5 \%$ and $25 \%$ of height level and height had significant effect and height had no significant effect on tracheid length of wood $(\mathrm{P}<0.05)$. The pattern of variation in tracheid length of wood, as a function of height in the stem, is shown in Table 2.

\begin{tabular}{|c|c|c|c|c|c|c|c|c|c|c|c|c|c|c|c|}
\hline $\begin{array}{c}\text { Height } \\
\text { Levels } \\
\text { of Total } \\
\text { Tree }\end{array}$ & $\mathrm{T}_{\mathrm{L}}$ & $T_{D}$ & $T_{L D}$ & $2 T_{W T}$ & $S_{R}$ & $F_{R}$ & $R_{R}$ & $D_{O}$ & $D_{B}$ & $B_{L}$ & $B_{R}$ & $B_{T}$ & $B_{V}$ & $\begin{array}{c}\mathrm{MC} \\
(\max )\end{array}$ & C \\
\hline \multirow[t]{2}{*}{$50 \%$} & $\begin{array}{c}4.8 \\
a\end{array}$ & $\underset{a}{61.7}$ & $\underset{a}{42.6}$ & $\underset{a}{19.1}$ & $\underset{a}{78.8}$ & $\underset{a}{69.0}$ & $\underset{a}{45.2}$ & $\underset{a}{0.45}$ & $\underset{a}{0.41}$ & $\underset{\mathrm{a}}{0.44}$ & $\underset{a}{3.03}$ & $\underset{\mathrm{a}}{5.83}$ & $\underset{a}{8.99}$ & 155.62 & 69.99 \\
\hline & 0.9 & 9.0 & 7.0 & 2.6 & 12.9 & 2.6 & 5.7 & 0.03 & 0.02 & 0.35 & 0.50 & 0.93 & 1.14 & 12.31 & 1.83 \\
\hline \multirow{2}{*}{$25 \%$} & $\begin{array}{c}4.9 \\
a\end{array}$ & $\underset{a}{63.2}$ & $\underset{\mathrm{a}}{44.2}$ & $\underset{a}{19.1}$ & $\underset{a}{77.4}$ & $\underset{a}{69.4}$ & $\underset{a}{44.6}$ & $\begin{array}{c}0.47 \\
a\end{array}$ & $\begin{array}{c}0.42 \\
a\end{array}$ & $\underset{\mathrm{ab}}{0.56}$ & $\begin{array}{c}3.11 \\
a\end{array}$ & $\underset{a}{6.08}$ & $\underset{\mathrm{ab}}{9.51}$ & 145.69 & $\underset{b}{68.87}$ \\
\hline & 1.2 & 10.8 & 9.5 & 2.0 & 11.9 & 3.7 & 7.8 & 0.03 & 0.02 & 0.38 & 0.60 & 0.76 & 0.98 & 11.70 & 1.81 \\
\hline \multirow{2}{*}{$5 \%$} & $\underset{a}{5.0}$ & $\underset{a}{64.7}$ & $\underset{\mathrm{a}}{44.7}$ & 20.0 & 77.6 & $\underset{a}{68.5}$ & $\underset{\mathrm{a}}{46.9}$ & 0.50 & 0.45 & 0.96 & $\underset{\mathrm{a}}{3.24}$ & $\underset{a}{6.12}$ & 10.17 & $\underset{a}{133.33}$ & $\underset{a}{66.69}$ \\
\hline & 1.2 & 13.3 & 11.3 & 3.4 & 12.7 & 5.2 & 12.1 & 0.03 & 0.02 & 0.70 & 0.69 & 1.24 & 1.04 & 13.53 & 1.86 \\
\hline
\end{tabular}

Table 2. Variation in Biometrical and Physical Properties of Wood in Longitudinal Direction at Different Height Levels within the Stem of C. sempervirens L.

Values are mean \pm standard deviation. Results with different letters are significantly different (Duncan's test). $T_{L}, T_{D}, T_{L D}, T_{W T}, S_{R}, F_{R}, R_{R}, D_{O}, D_{B}, B_{L}, B_{R}, B_{T}, B_{V}, M C$ (max), and $C$ are mean tracheid length $(\mathrm{mm})$, tracheid diameter $(\mu \mathrm{m})$, tracheid lumen diameter $(\mu \mathrm{m})$, tracheid wall thickness $(\mu \mathrm{m})$, slenderness ratio, flexibility ratio $(\%)$, Runkel ratio $(\%)$, oven-dry $\left(\mathrm{gr} / \mathrm{cm}^{3}\right)$ and basic density $\left(\mathrm{g} / \mathrm{cm}^{3}\right)$, longitudinal $(\%)$, radial $(\%)$, tangential $(\%)$, and volume (\%) shrinkage, maximum moisture content $(\%)$, and porosity (\%) respectively. 
Within-tree tracheid length of wood at each height level decreased from the base upwards; however, tracheid length of C. sempervirens L. wood was the highest at $5 \%$ of total tree height. Within the samples, at the same height level, tracheid length of wood increased from the pith to the bark (Table 3 ).

Table 3. Variation in Biometrical Property of Wood in Radius and Longitudinal Directions, According to Stem Height and Samples from the Pith to the Bark in C. sempervirens L.

\begin{tabular}{|c|c|c|c|c|c|c|c|c|c|c|}
\hline $\begin{array}{l}\text { Height Levels } \\
\text { of Total Tree }\end{array}$ & \multicolumn{10}{|c|}{ Samples from the Pith to the Bark } \\
\hline$\overline{T_{L}(\mathrm{~mm})}$ & 1 & 2 & 3 & 4 & 5 & 6 & 7 & 8 & 9 & 10 \\
\hline $50 \%$ & $4.5^{\mathrm{a}}$ & $4.2^{\mathrm{a}}$ & $4.2^{a}$ & $\begin{array}{c}4.7^{\mathrm{a}} \\
0.4\end{array}$ & $4.2^{\mathrm{a}}$ & $4.6^{a}$ & $4.9^{a}$ & $5.5^{\mathrm{a}}$ & $5.7^{\mathrm{a}}$ & $5.8^{a}$ \\
\hline $25 \%$ & $\begin{array}{c}4.1^{\mathrm{ab}} \\
1.2\end{array}$ & $\begin{array}{l}3.9^{\mathrm{a}} \\
1.2\end{array}$ & $\begin{array}{c}4.3^{\mathrm{ab}} \\
0.7\end{array}$ & $\begin{array}{c}4.8^{\mathrm{ab}} \\
1.4\end{array}$ & $\begin{array}{c}1.0^{\mathrm{ab}} \\
0.9\end{array}$ & $\begin{array}{c}0.1 \\
4.8^{\mathrm{ab}} \\
0.6\end{array}$ & $\begin{array}{c}5.1^{\mathrm{ab}} \\
1.8\end{array}$ & $\begin{array}{c}0.8^{\mathrm{ab}} \\
0.6\end{array}$ & $\begin{array}{c}1.1^{\mathrm{ab}} \\
0.7\end{array}$ & $\begin{array}{l}1.3^{\mathrm{b}} \\
1.7\end{array}$ \\
\hline $5 \%$ & $\begin{array}{l}3.4^{a} \\
1.3 \\
\end{array}$ & $\begin{array}{c}4.2^{\mathrm{ab}} \\
1.2 \\
\end{array}$ & $\begin{array}{c}4.2^{\mathrm{ab}} \\
0.3\end{array}$ & $\begin{array}{c}5.2^{\mathrm{ab}} \\
0.4\end{array}$ & $\begin{array}{c}5.2^{\mathrm{ab}} \\
1.3\end{array}$ & $\begin{array}{c}5.4^{\mathrm{ab}} \\
1.2\end{array}$ & $\begin{array}{c}5.4^{\mathrm{ab}} \\
0.7^{2}\end{array}$ & $\begin{array}{c}5.6^{\mathrm{b}} \\
0.9\end{array}$ & $\begin{array}{c}5.2^{\mathrm{ab}} \\
1.3\end{array}$ & $\begin{array}{l}6.1^{\mathrm{b}} \\
1.3\end{array}$ \\
\hline$\overline{T_{D}(\mu \mathrm{m})}$ & 1 & 2 & 3 & 4 & 5 & 6 & 7 & 8 & 9 & 10 \\
\hline $50 \%$ & $\begin{array}{l}66.9^{a} \\
17.2^{a}\end{array}$ & $\begin{array}{l}61.9^{a} \\
13.2^{a}\end{array}$ & $\begin{array}{c}59.2^{\mathrm{a}} \\
12.2^{2}\end{array}$ & $\begin{array}{c}59.9^{\mathrm{a}} \\
3.3^{2}\end{array}$ & $\begin{array}{c}60.8^{\mathrm{a}} \\
7.5\end{array}$ & $\begin{array}{c}60.9^{a} \\
9.0^{a}\end{array}$ & $\begin{array}{c}64.2^{\mathrm{a}} \\
7.6\end{array}$ & $\begin{array}{c}60.9^{a} \\
5.5^{a}\end{array}$ & $\begin{array}{c}62.1^{\mathrm{a}} \\
13.2^{2}\end{array}$ & $\begin{array}{c}60.1^{a} \\
8.9^{a}\end{array}$ \\
\hline $25 \%$ & $\begin{array}{c}64.3^{a} \\
7.2\end{array}$ & $\begin{array}{c}58.4^{\mathrm{a}} \\
14.0\end{array}$ & $\begin{array}{l}63.3^{\mathrm{a}} \\
7.6\end{array}$ & $\begin{array}{c}61.1^{a} \\
16.2\end{array}$ & $\begin{array}{c}66.9^{a} \\
10.1\end{array}$ & $\begin{array}{c}58.1^{\mathrm{a}} \\
6.3^{2}\end{array}$ & $\begin{array}{c}56.3^{a} \\
8.5\end{array}$ & $\begin{array}{l}59.4^{\mathrm{a}} \\
5.0\end{array}$ & $\begin{array}{c}70.1^{\mathrm{a}} \\
12.4\end{array}$ & $\begin{array}{l}74.6^{\mathrm{a}} \\
16.0\end{array}$ \\
\hline $5 \%$ & $\begin{array}{c}49.5^{a} \\
15.8\end{array}$ & $\begin{array}{c}58.1^{\mathrm{a}} \\
9.2^{2}\end{array}$ & $\begin{array}{c}63.6^{\mathrm{a}} \\
11.4\end{array}$ & $\begin{array}{c}67.1^{\mathrm{a}} \\
9.5\end{array}$ & $\begin{array}{l}68.2^{\mathrm{a}} \\
14.2\end{array}$ & $\begin{array}{l}68.4^{\mathrm{a}} \\
23.5\end{array}$ & $\begin{array}{c}65.9^{a} \\
8.8^{a}\end{array}$ & $\begin{array}{c}62.1^{\mathrm{a}} \\
4.7^{2}\end{array}$ & $\begin{array}{c}70.6^{\mathrm{a}} \\
16.4\end{array}$ & $\begin{array}{c}73.7^{a} \\
15.0\end{array}$ \\
\hline$\overline{T_{L D}(\mu \mathrm{m})}$ & 1 & 2 & 3 & 4 & 5 & 6 & 7 & 8 & 9 & 10 \\
\hline $50 \%$ & $\begin{array}{c}47.7^{a} \\
12.6\end{array}$ & $\begin{array}{l}42.1^{\mathrm{a}} \\
8.5\end{array}$ & $\begin{array}{c}40.5^{a} \\
8.5\end{array}$ & $\begin{array}{c}40.9^{a} \\
2.1\end{array}$ & $\begin{array}{c}42.5^{a} \\
4.6\end{array}$ & $\begin{array}{c}42.6^{a} \\
7.8\end{array}$ & $\begin{array}{c}44.4^{a} \\
5.4\end{array}$ & $\begin{array}{c}41.9^{a} \\
4.5\end{array}$ & $\begin{array}{c}42.6^{a} \\
11.6\end{array}$ & $\begin{array}{c}40.9^{a} \\
8.6\end{array}$ \\
\hline $25 \%$ & $\begin{array}{c}45.6^{\mathrm{a}} \\
6.0^{\mathrm{n}}\end{array}$ & $\begin{array}{c}40.9^{a} \\
13.2\end{array}$ & $\begin{array}{c}45.1^{\mathrm{a}} \\
6.8\end{array}$ & $\begin{array}{c}42.5^{a} \\
13.7\end{array}$ & $\begin{array}{c}46.9^{a} \\
10.6\end{array}$ & $\begin{array}{c}39.4^{a} \\
6.1\end{array}$ & $\begin{array}{c}38.5^{\mathrm{a}} \\
8.5\end{array}$ & $\begin{array}{c}39.8^{a} \\
2.8\end{array}$ & $\begin{array}{c}49.9^{\mathrm{a}} \\
12.3\end{array}$ & $\begin{array}{l}53.0^{\mathrm{a}} \\
13.3\end{array}$ \\
\hline $5 \%$ & $\begin{array}{l}31.6^{a} \\
14.6\end{array}$ & $\begin{array}{c}39.6^{a} \\
6.6\end{array}$ & $\begin{array}{c}43.7^{\mathrm{a}} \\
10.0\end{array}$ & $\begin{array}{c}46.2^{a} \\
8.2\end{array}$ & $\begin{array}{c}49.6^{\mathrm{a}} \\
13.0\end{array}$ & $\begin{array}{c}48.1^{\mathrm{a}} \\
21.1^{4}\end{array}$ & $\begin{array}{c}45.3^{a} \\
5.3^{a}\end{array}$ & $\begin{array}{c}43.6^{\mathrm{a}} \\
4.7\end{array}$ & $\begin{array}{c}49.9^{a} \\
13.5\end{array}$ & $\begin{array}{c}49.6^{a} \\
12.3\end{array}$ \\
\hline $2 T_{W T}(\mu \mathrm{m})$ & 1 & 2 & 3 & 4 & 5 & 6 & 7 & 8 & 9 & 10 \\
\hline $50 \%$ & $\begin{array}{c}19.1^{\mathrm{a}} \\
4.6\end{array}$ & $\begin{array}{c}19.8^{a} \\
4.7\end{array}$ & $\begin{array}{c}18.7^{\mathrm{a}} \\
3.7^{\mathrm{n}}\end{array}$ & $\begin{array}{c}18.9^{a} \\
1.8\end{array}$ & $\begin{array}{c}18.3^{\mathrm{a}} \\
3.3^{2}\end{array}$ & $\begin{array}{c}18.3^{\mathrm{a}} \\
1.2\end{array}$ & $\begin{array}{c}19.8^{\mathrm{a}} \\
3.1\end{array}$ & $\begin{array}{c}19.0^{\mathrm{a}} \\
3.4\end{array}$ & $\begin{array}{c}19.4^{\mathrm{a}} \\
1.6\end{array}$ & $\begin{array}{c}19.2^{a} \\
1.1\end{array}$ \\
\hline $25 \%$ & $\begin{array}{c}18.7^{\mathrm{ab}} \\
1.3\end{array}$ & $\begin{array}{c}17.4^{\mathrm{a}} \\
0.9^{2}\end{array}$ & $\begin{array}{c}18.1^{\mathrm{ab}} \\
0.9\end{array}$ & $\begin{array}{c}18.6^{\text {ab }} \\
2.5\end{array}$ & $\begin{array}{c}20.0^{\mathrm{ab}} \\
2.1\end{array}$ & $\begin{array}{c}18.7^{\mathrm{ab}} \\
0.3\end{array}$ & $\begin{array}{c}17.8^{\mathrm{a}} \\
0.0^{\circ}\end{array}$ & $\begin{array}{c}19.6^{\mathrm{ab}} \\
3.3\end{array}$ & $\begin{array}{c}20.2^{\mathrm{ab}} \\
2.0\end{array}$ & $\begin{array}{c}21.6^{b} \\
2.9\end{array}$ \\
\hline $5 \%$ & $\begin{array}{c}17.9^{a} \\
2.3^{a}\end{array}$ & $\begin{array}{c}18.5^{a} \\
3.0\end{array}$ & $\begin{array}{c}19.9^{\mathrm{a}} \\
1.9^{-}\end{array}$ & $\begin{array}{c}20.9^{a} \\
4.7^{2}\end{array}$ & $\begin{array}{c}18.6^{\mathrm{a}} \\
1.6\end{array}$ & $\begin{array}{l}20.3^{a} \\
2.4\end{array}$ & $\begin{array}{c}20.6^{\mathrm{a}} \\
3.8^{2}\end{array}$ & $\begin{array}{c}18.5^{\mathrm{a}} \\
0.1\end{array}$ & $\begin{array}{c}20.7^{a} \\
2.9\end{array}$ & $\begin{array}{c}24.1^{\mathrm{a}} \\
7.0\end{array}$ \\
\hline$\overline{S_{R}}$ & 1 & 2 & 3 & 4 & 5 & 6 & 7 & 8 & 9 & 10 \\
\hline $50 \%$ & $\begin{array}{c}67.7^{a} \\
1.0\end{array}$ & $\begin{array}{c}68.4^{a} \\
3.9^{a}\end{array}$ & $\begin{array}{c}72.4^{\mathrm{a}} \\
10.5\end{array}$ & $\begin{array}{c}78.8^{\text {ab }} \\
8.1\end{array}$ & $\begin{array}{c}68.8^{\mathrm{a}} \\
7.4\end{array}$ & $\begin{array}{c}75.1^{\text {ab }} \\
6.0\end{array}$ & $\begin{array}{c}76.9^{\mathrm{ab}} \\
12.6\end{array}$ & $\begin{array}{c}90.9^{\mathrm{bc}} \\
18.8\end{array}$ & $\begin{array}{c}92.0^{\mathrm{bc}} \\
5.3\end{array}$ & $\begin{array}{c}96.8^{c} \\
6.0^{\circ}\end{array}$ \\
\hline $25 \%$ & $\begin{array}{c}63.6^{\mathrm{a}} \\
11.3\end{array}$ & $\begin{array}{c}66.8^{\mathrm{ab}} \\
3.8^{2}\end{array}$ & $\begin{array}{c}68.3^{\text {ab }} \\
3.1\end{array}$ & $\begin{array}{c}78.5^{\mathrm{abc}} \\
4.1\end{array}$ & $\begin{array}{c}73.9^{\mathrm{abc}} \\
2.9\end{array}$ & $\begin{array}{c}82.3^{\text {abc }} \\
5.3\end{array}$ & $\begin{array}{l}88.4^{c} \\
20.0\end{array}$ & $\begin{array}{c}80.2^{\text {abc }} \\
7.7\end{array}$ & $\begin{array}{c}88.3^{c} \\
16.5\end{array}$ & $\begin{array}{c}83.6^{b c} \\
6.9\end{array}$ \\
\hline $5 \%$ & $\begin{array}{c}68.9^{a} \\
3.1\end{array}$ & $\begin{array}{c}71.2^{a} \\
9.0\end{array}$ & $\begin{array}{c}68.1^{\mathrm{a}} \\
14.0\end{array}$ & $\begin{array}{c}78.9^{a} \\
12.4\end{array}$ & $\begin{array}{c}76.7^{a} \\
20.2\end{array}$ & $\begin{array}{c}83.4^{a} \\
25.5\end{array}$ & $\begin{array}{c}82.2^{a} \\
8.1\end{array}$ & $\begin{array}{c}90.1^{\mathrm{a}} \\
8.4^{2}\end{array}$ & $\begin{array}{c}74.3^{\mathrm{a}} \\
5.9\end{array}$ & $\begin{array}{c}82.1^{a} \\
1.1\end{array}$ \\
\hline
\end{tabular}


Table 3 (continued). Variation in Biometrical Property of Wood in Radius and Longitudinal Directions, According to Stem Height and Samples from the Pith to the Bark in C. sempervirens L.

\begin{tabular}{|c|c|c|c|c|c|c|c|c|c|c|}
\hline $\begin{array}{l}\text { Height Levels } \\
\text { of Total Tree }\end{array}$ & \multicolumn{10}{|c|}{ Samples from the Pith to the Bark } \\
\hline$F_{R}(\%)$ & 1 & 2 & 3 & 4 & 5 & 6 & 7 & 8 & 9 & 10 \\
\hline $50 \%$ & $\begin{array}{c}71.3^{a} \\
0.8^{a}\end{array}$ & $\begin{array}{c}68.1^{\mathrm{a}} \\
0.7^{\mathrm{n}}\end{array}$ & $\begin{array}{c}68.4^{a} \\
0.8^{a}\end{array}$ & $\begin{array}{c}68.4^{a} \\
1.8\end{array}$ & $\begin{array}{c}70.0^{a} \\
2.3^{a}\end{array}$ & $\begin{array}{c}69.7^{a} \\
2.8^{a}\end{array}$ & $\begin{array}{c}69.2^{a} \\
2.8\end{array}$ & $\begin{array}{c}68.8^{a} \\
4.4^{a}\end{array}$ & $\begin{array}{c}68.2^{a} \\
3.8\end{array}$ & $\begin{array}{c}67.6^{a} \\
4.9\end{array}$ \\
\hline $25 \%$ & $\begin{array}{c}70.8^{a} \\
1.4\end{array}$ & $\begin{array}{c}69.3^{\mathrm{a}} \\
5.5^{\mathrm{N}}\end{array}$ & $\begin{array}{l}71.2^{a} \\
2.0\end{array}$ & $\begin{array}{c}68.9^{a} \\
3.9^{a}\end{array}$ & $\begin{array}{c}69.6^{\mathrm{a}} \\
6.0^{\circ}\end{array}$ & $\begin{array}{c}67.6^{\mathrm{a}} \\
2.9^{\circ}\end{array}$ & $\begin{array}{c}67.9^{a} \\
5.1\end{array}$ & $\begin{array}{c}67.1^{a} \\
3.4^{a}\end{array}$ & $\begin{array}{c}70.7^{\mathrm{a}} \\
5.1\end{array}$ & $\begin{array}{l}70.7^{a} \\
2.6^{a}\end{array}$ \\
\hline $5 \%$ & $\begin{array}{c}62.3^{a} \\
8.9^{a} \\
\end{array}$ & $\begin{array}{c}68.1^{a} \\
2.0^{a} \\
\end{array}$ & $\begin{array}{c}68.3^{a} \\
3.5^{3}\end{array}$ & $\begin{array}{c}68.9^{a} \\
5.8^{a}\end{array}$ & $\begin{array}{c}72.2^{a} \\
4.3^{a} \\
\end{array}$ & $\begin{array}{c}68.8^{a} \\
7.0^{a} \\
\end{array}$ & $\begin{array}{c}68.8^{\mathrm{a}} \\
2.4^{-}\end{array}$ & $\begin{array}{c}70.1^{a} \\
2.3^{a} \\
\end{array}$ & $\begin{array}{c}70.2^{a} \\
3.2^{2}\end{array}$ & $\begin{array}{c}67.1^{\mathrm{a}} \\
8.8^{-} \\
\end{array}$ \\
\hline$R_{R}(\%)$ & 1 & 2 & 3 & 4 & 5 & 6 & 7 & 8 & 9 & 10 \\
\hline $50 \%$ & $\begin{array}{c}40.3^{a} \\
1.7^{a}\end{array}$ & $\begin{array}{c}46.7^{\mathrm{a}} \\
1.5^{2}\end{array}$ & $\begin{array}{c}46.3^{a} \\
1.7\end{array}$ & $\begin{array}{c}46.3^{a} \\
3.8^{a}\end{array}$ & $\begin{array}{c}42.9^{a} \\
4.6^{a}\end{array}$ & $\begin{array}{c}43.6^{a} \\
5.8\end{array}$ & $\begin{array}{c}44.7^{\mathrm{a}} \\
5.8\end{array}$ & $\begin{array}{c}45.7^{a} \\
9.3^{a}\end{array}$ & $\begin{array}{c}47.0^{\mathrm{a}} \\
7.9^{\circ}\end{array}$ & $\begin{array}{c}48.5^{a} \\
11.3^{a}\end{array}$ \\
\hline $25 \%$ & $\begin{array}{c}41.3^{\mathrm{a}} \\
2.9^{\circ}\end{array}$ & $\begin{array}{c}45.0^{\mathrm{a}} \\
11.2^{2} \\
\end{array}$ & $\begin{array}{c}40.6^{\mathrm{a}} \\
3.9^{-}\end{array}$ & $\begin{array}{c}45.4^{\mathrm{a}} \\
8.2^{2} \\
\end{array}$ & $\begin{array}{c}44.4^{\mathrm{a}} \\
13.1 \\
\end{array}$ & $\begin{array}{c}48.2^{a} \\
6.2^{a}\end{array}$ & $\begin{array}{c}47.9^{\mathrm{a}} \\
11.4 \\
\end{array}$ & $\begin{array}{c}49.4^{\mathrm{a}} \\
7.8^{\mathrm{P}} \\
\end{array}$ & $\begin{array}{c}41.9^{a} \\
10.1 \\
\end{array}$ & $\begin{array}{c}41.5^{\mathrm{a}} \\
5.2^{2}\end{array}$ \\
\hline $5 \%$ & $\begin{array}{l}62.7^{a} \\
22.9^{a}\end{array}$ & $\begin{array}{c}47.0^{\mathrm{a}} \\
4.2^{2}\end{array}$ & $\begin{array}{l}46.6^{a} \\
7.3^{a}\end{array}$ & $\begin{array}{l}45.9^{a} \\
12.7^{a}\end{array}$ & $\begin{array}{c}38.8^{a} \\
8.5^{a}\end{array}$ & $\begin{array}{l}46.4^{a} \\
14.7^{a}\end{array}$ & $\begin{array}{c}45.4^{a} \\
5.2^{a}\end{array}$ & $\begin{array}{c}42.7^{a} \\
4.6\end{array}$ & $\begin{array}{c}42.8^{a} \\
6.7^{a}\end{array}$ & $\begin{array}{l}50.8^{a} \\
20.5^{a}\end{array}$ \\
\hline
\end{tabular}

Values are mean \pm standard deviation. Results with different letters are significantly different (Duncan's test). $T_{L}, T_{D}, T_{L D}, T_{W T}, S_{R}, F_{R}$, and $R_{R}$, are mean tracheid length, tracheid diameter, tracheid lumen diameter, tracheid wall thickness, slenderness ratio, flexibility ratio, and Runkel ratio, respectively.

The results of ANOVA indicated that radial position and height had no significant effect on tracheid diameter and lumen diameter of wood $(\mathrm{P}<0.05)$. The pattern of variation in tracheid diameter and lumen diameter of wood, as a function of height in the stem, is shown in Table 2.

Within-tree tracheid diameter and lumen diameter of wood at each height level decreased from the base upwards; however, tracheid diameter and lumen diameter of $C$. sempervirens L. wood was the highest at 5\% of total tree height. Within the samples, at the same height level, tracheid diameter and lumen diameter of wood increased from the pith to the bark, with the exception of the $50 \%$ of height level (Table 3 ).

The ANOVA results also indicated that radial position had significant effect on tracheid wall thickness of wood at the $25 \%$ of height level $(\mathrm{P}<0.05)$, but height had no significant effect on tracheid wall thickness of wood. The pattern of variation in tracheid wall thickness of wood, as a function of height in the stem, is shown in Table 2.

Within-tree tracheid wall thickness of wood at each height level decreased from the base upwards; however, tracheid wall thickness of $C$. sempervirens L. wood was the highest at $5 \%$ of total tree height. Within the samples, at the same height level, tracheid wall thickness of wood decreased from the pith to the bark, with the exception of $50 \%$ of height level (Table 3).

The ANOVA results indicated that radial position at $25 \%$ and $50 \%$ of height level had significant effect and height had no significant effect on slenderness ratio of tracheid $(\mathrm{P}<0.05)$. The pattern of variation in slenderness ratio of tracheid, as a function of height in the stem, is shown in Table 2. 
Within-tree slenderness ratio of tracheid at each height level increased from the base upwards; however, slenderness ratio of $C$. sempervirens L. tracheid was the highest at $50 \%$ of total tree height. Within the samples, at the same height level, slenderness ratio of tracheid increased from the pith to the bark (Table 3 ).

The results of ANOVA indicated that radial position and height had no significant effect on flexibility and Runkell ratio of tracheid $(\mathrm{P}<0.05)$. The pattern of variation in flexibility and Runkell ratio of tracheid, as a function of height in the stem, is shown in Table 2.

Within-tree flexibility and Runkell ratio of tracheid at each height level increased and decreased from the base upwards respectively; however, flexibility and Runkell ratio of C. sempervirens L. tracheid were the highest at 5\% of total tree height. Within the samples, at the same height level, flexibility and Runkell ratio of tracheid increased from the pith to the bark at $5 \%$ and $50 \%$ of height level, respectively (Table 3 ).

The results of ANOVA indicated that height had significant effect and radial position had no significant effect on oven-dry and basic density of wood $(\mathrm{P}<0.05)$. The pattern of variation in oven-dry and basic density of wood, as a function of height in the stem, is shown in Table 2.

Within-tree dry and basic density of wood at each height level decreased from the base upwards; however, oven-dry and basic density of $C$. sempervirens L. wood was the highest at $5 \%$ of total tree height. Within the samples, at the same height level, oven-dry and basic density of wood increased from the pith to the bark (Table 4).

ANOVA showed that the radial position and height had significant effects on longitudinal shrinkage $(\mathrm{P}<0.05)$. The pattern of variation in longitudinal shrinkage, as a function of the height in the stem, is shown in Table 2. Within-tree longitudinal shrinkage at each height level decreased along the stem, from the base upwards; however, wood longitudinal shrinkage was the highest at $5 \%$ of total tree height.

As shown in Table 4, within the samples, at the same height levels, longitudinal shrinkage decreased from the pith outwards.

ANOVA showed that the radial position had significant effects on tangential, volume at total height level, and radial shrinkage at $5 \%$ and $25 \%$ of height level $(\mathrm{P}<$ $0.05)$. The pattern of variation in radial, tangential, and volume shrinkage, as a function of the height in the stem, is shown in Table 2. Within-tree radial, tangential, and volume shrinkage at each height level decreased along the stem, from the base upwards; however, wood radial, tangential, and volume shrinkage was the highest at 5\% of total tree height. Also height had no significant effects on radial and tangential shrinkage, but had significant effects on volume shrinkage.

As shown in Table 4, within the samples, at the same height levels, radial, tangential, and volume shrinkage increased from the pith outwards. ANOVA indicated that radial position had no significant effect and height position had significant effect on maximum moisture content $(\mathrm{P}<0.05)$. The pattern of variation in maximum moisture content, as a function of height in the stem, is shown in Table 2. Within-tree maximum moisture content at each height level increased from the base upwards; however, for $C$. sempervirens L. the maximum moisture content was highest at $50 \%$ of total tree height. Within the samples, at the same height level maximum moisture content increased from the pith to the bark exceptionally $5 \%$ of height level (Table 4 ). 
Table 4. Variation in Physical Property of Wood in Radius and Longitudinal Directions, According to Stem Height and Samples from the Pith to the Bark in C. sempervirens L.

\begin{tabular}{|c|c|c|c|c|c|}
\hline $\begin{array}{l}\text { Height Levels } \\
\text { of Total Tree }\end{array}$ & \multicolumn{5}{|c|}{ Samples from the Pith to the Bark } \\
\hline$D_{O}\left(\mathrm{~g} / \mathrm{cm}^{3}\right)$ & 1 & 2 & 3 & 4 & 5 \\
\hline $50 \%$ & $0.44^{\mathrm{a}} 0.03$ & $0.45^{\mathrm{a}} 0.03$ & $0.46^{\mathrm{a}} 0.04$ & - & - \\
\hline $25 \%$ & $0.45^{\mathrm{a}} 0.02$ & $0.47^{\mathrm{a}} 0.04$ & $0.47^{\mathrm{a}} 0.02$ & $0.48^{a} 0.03$ & - \\
\hline $5 \%$ & $0.50^{a} 0.01$ & $0.50^{\mathrm{a}} 0.02$ & $0.49^{a} 0.05$ & $0.49^{a} 0.02$ & $0.51^{\mathrm{a}} 0.04$ \\
\hline$D_{B}\left(\mathrm{~g} / \mathrm{cm}^{3}\right)$ & 1 & 2 & 3 & 4 & 5 \\
\hline $50 \%$ & $0.41^{\mathrm{a}} 0.03$ & $0.41^{\mathrm{a}} 0.02$ & $0.41^{\mathrm{a}} 0.03$ & - & - \\
\hline $25 \%$ & $0.41^{\mathrm{a}} 0.02$ & $0.42^{a} 0.04$ & $0.43^{a} 0.02$ & $0.43^{a} 0.03$ & - \\
\hline $5 \%$ & $0.45^{a} 0.01$ & $0.46^{a} 0.02$ & $0.44^{\mathrm{a}} 0.04$ & $0.44^{a} 0.02$ & $0.46^{a} 0.04$ \\
\hline$B_{L}(\%)$ & 1 & 2 & 3 & 4 & 5 \\
\hline $50 \%$ & $0.89^{b} 0.09$ & $0.28^{a} 0.16$ & $0.16^{a} 0.12$ & - & - \\
\hline $25 \%$ & $0.89^{\mathrm{C}} 0.25$ & $0.79^{b c} 0.20$ & $0.39^{\mathrm{ab}} 0.39$ & $0.15^{\mathrm{a}} 0.00$ & - \\
\hline $5 \%$ & $1.46^{\mathrm{b}} 0.50$ & $1.80^{\mathrm{b}} 0.31$ & $0.69^{a} 0.27$ & $0.45^{\mathrm{a}} 0.10$ & $0.23^{a} 0.08$ \\
\hline$B_{R}(\%)$ & 1 & 2 & 3 & 4 & 5 \\
\hline $50 \%$ & $2.58^{\mathrm{a}} 0.43$ & $3.18^{\mathrm{a}} 0.38$ & $3.33^{a} 0.46$ & - & - \\
\hline $25 \%$ & $2.55^{\mathrm{a}} 0.04$ & $2.89^{\mathrm{ab}} 0.38$ & $3.25^{\mathrm{ab}} 0.78$ & $3.73^{b} 0.27$ & - \\
\hline $5 \%$ & $\begin{array}{l}2.71^{a} \\
0.84\end{array}$ & $\begin{array}{l}2.71^{a} \\
0.23\end{array}$ & $\begin{array}{c}3.23^{\mathrm{ab}} \\
0.55\end{array}$ & $\begin{array}{c}3.66^{\mathrm{ab}} \\
0.74\end{array}$ & $\begin{array}{l}3.88^{b} \\
0.23\end{array}$ \\
\hline$B_{T}(\%)$ & 1 & 2 & 3 & 4 & 5 \\
\hline $50 \%$ & $4.75^{\mathrm{a}} 0.69$ & $6.35^{\mathrm{b}} 0.59$ & $6.39^{b} 0.18$ & - & - \\
\hline $25 \%$ & $5.14^{\mathrm{a}} 0.18$ & $6.05^{\mathrm{ab}} 0.65$ & $6.44^{b} 0.73$ & $6.70^{b} 0.32$ & - \\
\hline $5 \%$ & $4.70^{\mathrm{a}} 0.91$ & $4.99^{\mathrm{a}} 0.65$ & $6.71^{\mathrm{b}} 0.85$ & $7.21^{\mathrm{b}} 0.47$ & $7.01^{b} 0.46$ \\
\hline$B_{V}(\%)$ & 1 & 2 & 3 & 4 & 5 \\
\hline $50 \%$ & $7.72^{\mathrm{a}} 0.69$ & $9.60^{\mathrm{b}} 0.91$ & $9.63^{b} 0.57$ & - & - \\
\hline $25 \%$ & $8.39^{a} 0.32$ & $9.49^{\mathrm{ab}} 0.85$ & $9.85^{\mathrm{ab}} 1.08$ & $10.29^{b} 0.57$ & - \\
\hline $5 \%$ & $9.42^{\mathrm{ab}} 1.04$ & $9.23^{a} 0.56$ & $10.34^{\mathrm{ab}} 1.22$ & $11.00^{b} 0.67$ & $10.84^{\mathrm{ab}} 0.56$ \\
\hline$M C(\max )(\%)$ & 1 & 2 & 3 & 4 & 5 \\
\hline $50 \%$ & $150.41^{\text {a }} 14.80$ & $156.57^{\mathrm{a}} 10.56$ & $159.89^{\mathrm{a}} 14.35$ & - & - \\
\hline $25 \%$ & $145.11^{\mathrm{a}} 7.77$ & $143.64^{\mathrm{a}} 17.63$ & $147.93^{\mathrm{a}} 11.51$ & $146.08^{\mathrm{a}} 15.31$ & - \\
\hline $5 \%$ & $125.07^{\mathrm{a}} 6.80$ & $123.73^{\mathrm{a}} 10.94$ & $139.63^{\mathrm{a}} 18.62$ & $143.17^{\mathrm{a}} 4.14$ & $135.04^{\mathrm{a}} 1738$ \\
\hline$C(\%)$ & 1 & 2 & 3 & 4 & 5 \\
\hline $50 \%$ & $70.65^{a} 1.76$ & $69.99^{a} 1.77$ & $69.32^{a} 2.41$ & - & - \\
\hline $25 \%$ & $69.99^{a} 1.34$ & $68.87^{a} 2.53$ & $68.65^{a} 1.16$ & $67.99^{a} 2.31$ & - \\
\hline $5 \%$ & $66.87^{\mathrm{a}} 0.39$ & $66.65^{\mathrm{a}} 1.33$ & $67.09^{a} 3.36$ & $67.09^{a} 1.39$ & $65.75^{\mathrm{a}} 2.69$ \\
\hline
\end{tabular}

Values are mean \pm standard deviation. Results with different letters are significantly different (Duncan's test). $D_{O}, D_{B}, B_{L}, B_{R}, B_{T}, B_{V}, M C$ (max), and $C$ are mean oven-dry and basic density, longitudinal, radial, tangential, and volume shrinkage, and maximum moisture content respectively. 
ANOVA showed that radial position had no significant effect and height position had significant effect on porosity $(\mathrm{P}<0.05)$. The pattern of variation in porosity, as a function of the height in the stem, is shown in Table 2. Within-tree porosity at each height level increased along the stem, from the base upwards; however, porosity was the highest at $50 \%$ of total tree height.

As shown in Table 4, within the samples, at the same height levels, porosity decreased from the pith outwards.

There has been little information on the mean values of dry and basic density, longitudinal, radial, tangential, and volume shrinkage, maximum moisture content, porosity, tracheid length, tracheid diameter, tracheid lumen diameter, tracheid wall thickness, slenderness ratio, flexibility ratio, and Runkel ratio and on the trend of variation along and across the stem of the studied traits of plantation cypress wood. The mean values of specific gravity, green density, tracheid length, and tracheid crosssectional dimensions of the trees of this study were somewhat greater than those reported previously by Uzielli and Berti (1979), Paraskevopoulou (1991), Bannister and Orman (1960), and Hasegava et al. (2010), who refer to a natural population and plantation material.

The increase in average tracheid length across discs is common to many tree species (Dinwoodie 1961). Generally, in conifers, there seems to be a decrease in specific gravity and tracheid length with an increase in height (Ishengoma et al. 1995; Kibblewhite 1984; Malan 1989; Muneri and Balodis 1998), and significant differences have been found at different sampling heights for ring width, latewood width, and latewood percentage (Malan 1989), where Malan (1989) found a decrease in tracheid length with height, he did find an increase from the ground level to about $15 \%$ of tree height before it decreased. This trend was also shown by Muneri and Balodis (1998).

According to the authors' observations from ring width of samples, the ring width in plantation cypress is low in the innermost rings $(2 \mathrm{~cm}, 4 \mathrm{~cm}$, and $6 \mathrm{~cm}$ of $5 \%, 25 \%$, and 50\% height levels respectively) and then increases from the pith outward until a maximum is reached with respect to rings $(4 \mathrm{~cm}, 6 \mathrm{~cm}$, and $8 \mathrm{~cm}$ at $5 \%, 25 \%$, and $50 \%$ of height levels respectively), after which it falls abruptly and extremely towards the bark (6 $\mathrm{cm}, 8 \mathrm{~cm}$, and $10 \mathrm{~cm}$ at $5 \%, 25 \%$, and $50 \%$ of height levels respectively), where with increase and decrease of ring width in conifers, earlywood is relatively constant, but latewood shows an increase and decrease. Thus, a considerable number of investigations have shown that there is a decrease in specific gravity with increasing ring width and greater ring width is associated with shorter tracheid length (Echols 1958; Panshin and De Zeeuw 1970). The studies performed on the annually ring of spruce (Dinwoodie 1961) and radiata pine (Nicholls and Dadswell 1962) have shown that tracheid length in earlywood is lower than latewood, falls to a minimum further out, and then increases in the final phase of season growth, where the value of length increase is between 12 to $20 \%$ of tracheid length in the initial of season growth.

In this study, the average values of intrinsic wood and tracheid characteristics such as $T_{D}, T_{L D}, T_{W T}, S_{R}, F_{R}(\%)$, and $R_{R}(\%)$ that were measured play an integral part in determining the quality of pulp or paper. It would appear that $T_{W T}$, especially of the latewood, specific gravity, and the Runkel ratio are best fiber dimension ratios to indicate of a number of handsheet properties. These attributes refer to the ratio between double 
the wall thicknesses and lumen diameter and could therefore be considered as traits in a breeding program, where they can account for 80 to $85 \%$ (Barefoot et al. 1964; Kibblewhite 1982) of the variation in the handsheet tear, and if this ratio is less than 1 , the collapsibility is most desirable.

Desirable values for density may be provided by either the $5 \%$ of height level and near the bark of trees, since the preference for high or low density in a species depends on the desired end-use (Dadswell and Nicholls 1959; Nicholls et al. 1963). Blair et al. (1975) reported that high-density wood is preferred for construction and furniture uses and it has generally been assumed to be preferable for pulping. However, if the main purpose is the conversion to sawn lumber, then high density will confer the best strength properties and high density should be the criterion when selecting for this feature. Research has shown that higher density species tend to have stronger timber than lower density species (Tsehaye et al. 1995b; Walker and Butterfield 1996).

Basic density is closely related to wood swelling and shrinkage (Newlin and Wilson 1919), where the total swelling and shrinkage $(R)$ accounted for 177 species of American woods via basic density $\left(D_{B}\right)$ according to the following formula: $R=28 D_{B}$, and a such closed relation accounted also for 131 Australian wood species with a 0.76 correlation coefficient at a 99.9\% confidence level (Kelsey 1956).

The results showed that volume shrinkage in cypress wood was maximal at the $5 \%$ of height level and the cross section bark-surface and minimal at the $50 \%$ of height level and close to the pith.

The juvenile wood with thinner cell walls and smaller cell dimensions is sometimes defined as a fixed number of annual rings from the pith outwards and a concern for utilization, particularly for fast-grown trees, which can be desirable for certain pulp and paper products, but the presence of juvenile wood with higher density near to the pith at $5 \%$ of height level than $50 \%$ of height level induces a greater variability in the raw material and greater longitudinal shrinkage.

Longitudinal shrinkage increased slightly downwards from the $50 \%$ of height level, whereas radial wood shrinkage was minimal at the stump height and at the $50 \%$ of height level. Tangential shrinkage was minimal at the $50 \%$ of height level and remained quite steady within the tree.

Simpson (1991) reported that the maximum moisture content in lumber is important because of its influence in controlling kiln-drying schedules. From a practical standpoint, when determining kiln schedules, the largest number of moisture samples should be selected from the slowest-drying material. Regarding the range of maximum moisture content in our sample, approximately 124 percent was found in the $5 \%$ of height level and in the near pith, whereas approximately 166 percent was found in the $50 \%$ of height level and in the near bark.

\section{CONCLUSIONS}

1. Significant differences in wood tracheid length and slenderness ratio existed between the radial position and height in C. sempervirens L. trees. 
2. There was a general trend in the radial and axial directions in C. sempervirens L. trees, in which wood tracheid dimensions and biometrical ratio varied from the pith to bark and from the base upwards.

3. Significant differences in wood density, and longitudinal, radial, tangential, and volumetric shrinkage existed between the radial position and height in C. sempervirens $\mathrm{L}$. trees.

4. There was a general trend in the radial and axial directions in C. sempervirens L. trees, in which wood density, radial, tangential, and volume shrinkage increased, from the pith to bark, with the exception of longitudinal shrinkage, and from the base upwards.

5. Significant differences in wood porosity and maximum moisture content existed in the height position, where porosity is linearly and inversely related to wood maximum moisture content and density respectively.

\section{REFERENCES CITED}

ASTM D 143-94 (2000). Standard Test Methods for Small Clear Specimens of Timber, Philadelphia, PA., USA.

Bannister, M. H., and Orman, H. R. (1960). "Cupressus lusitanica as a potential timber tree for New Zealand," New Zealand Journal Forestry 8, 203B217.

Barefoot, A. C., Hitching, R. G., and Ellwood, E. L. (1964). "Wood characteristics and Kraft paper properties of four selected Loblolly pines. Effect of fiber morphology under identical cooking conditions," Tappi 47(6), 343-355.

Blair, R. L., Zobel, B. J., and Barker, J. A. (1975). "Predictions of gain in pulp yield and tear strength in young loblolly pine through genetic increases in wood density," Tappi 58(1), 89-91.

Brazier, J. D., and Howell, R. S. (1979). "The use of a breast height core for estimating selected whole tree properties of Sitka spruce," Forestry 52(2), 177-185.

Brown, J. R. (1961). "Clonal variation in specific gravity in Populus tremuloides," MSc Thesis. State Coll. of forestry, Syracuse.

Cave, I. D., and Walker, J. C. F. (1994). "Stiffness of wood in fast-grown plantation softwoods: the influence of microfibril angle," Forest Products Journal 44(5), 43-48.

Dadswell, H. E., and Nicholls, J. W. P. (1959). "Assessment of wood qualities for tree breeding," I. In Pinus elliottii vat. elliottii from Queensland. Div. Forest Prod. Tech. Paper No. 4, CSIRO, Australia.

Deresse, T., Shepard, R. K., and Rice, R. W. (2002). "Longitudinal shrinkage, kiln-drying defects, and lumber grade recovery of red pine (Pinus resinasa Ait.) from a 125-yearold natural stand and a 57-yearold plantation," Forest Prod. J. 52(5), 88-93.

Dinwoodie, J. M. (1961). "Tracheid and fiber length in timber- A review of literature," Forestry 34 (2), 125-144.

Echols, R. M. (1958). "Variation in tracheid length and wood density in geographic races of scots pine," Yale University School of Forestry Technical Bulletin No. 64.

Einspher, D. W., Benson, M. K., and Peckham, J. R. (1967). "Variation and heritability of wood and growth characteristics of five-year old quaking aspen," Genet. Phys. Notes Inst. pap. chem. no 1. Applcton. 
Elliot, G. K. (1970). “Wood density in conifers," Technical communication No. 8, Commonwealth Forestry Bureau, Oxford, England.

Franklin, F. L. (1946). "A rapid method for softening wood for microtome sectioning," Trop. Woods Yale Univ. Sch. For. 88, 35-36.

Fuglem, G., Sabourin, M. J., and Lundqvist, S. -O. (2003). "Influence of spruce wood properties on thermo-mechanical pulping-pilot scale results," Conference proceeding, International Mechanical Pulping Conference, Quebec City 2-5 June.

Harvald, C., and Olesen, P. O. (1987). "The variation of the basic density within the juvenile wood of Sitka spruce (Picea sitchensis)," Scandinavian Journal of Forest Research 2, 525-537.

Haygreen, J. G., and Bowyer, J. L. (1982). "Forest products and wood science: An introduction," The Iowa State University Press, Ames, U.S.A., pp. 105-135.

Herritsch, A. (2007). "Investigations on wood stability and related properties of radiata pine," $\mathrm{PhD}$ thesis, University of Canterbury, Christchurch, New Zealand.

Ishengoma, R. C., Gillah, P. R., and Iddi, S. (1995). "Basic density, tracheid length and strength properties of juvenile and mature wood of Pinus patula grown in Tanzania," South African Forestry Journal 172, 19-23.

Jyske, T., Mäkinen, H. and Saranpää, P. (2008). "Wood density within Norway spruce stems," Silva, Fennica 42(3), 439-455. FI-01301 Vantaa, Finland.

Kelsey, K. E. (1956). "The shrinkage intersection point. Its significance and the method of its determination," Forest Prod. J. 6 (10), 411-417.

Kibblewhite, R. P. (1982). "The qualities of radiata pine paper making fibers," Appita 35 (4), 289-298.

Kibblewhite, R. P. (1984). "Pinus radiata wood residue qualities and some utilization options," New Zealand Journal of Forestry Science 14 (3), 382-394.

Kibblewhite, R. P. (1999). "Designer fibers for improved papers through exploiting genetic variation in wood microstructure," Proceedings, PIRA Conference, B 252, p. $1-11$.

Kilger, R., Johansson, M., and Perstorper, M. (2003). "Distortion of Norway spruce timber, Part 3: Modelling bow and spring," Holz als Roh- und Werkstoff 61, 241-250.

Koch, P. (1985). "Utilization of hardwoods growing on southern pine sites," Vol. 1, USDA For. Serv. Handb. 605, 465-548.

Malan, F. S. (1989). "Wood property variation in six Pinus radiata (D.Don) trees grown in the Jonkershoek State Forest," South African Forestry Journal 151, 39-45.

Muneri, A., and Balodis, V. (1998). "Variation in wood density and tracheid length in Pinus patula grown in Zimbabwe," South African Forestry Journal 182, 41-50.

Newlin, J., and Wilson, T. R. C., (1919). "The relationship of shrinkage and strength properties of wood to its specific gravity," Bull. No. 676. U.S. Department of Agriculture, Forest Service, Washington, DC.

Nicholls, J. W. P., and Dadswell, H. E. (1962). "Tracheid length in Pinus radiata D.Don," CSIRO Div., For. Prod. Techn. Paper No. 24.

Nicholls, J. W. P., Dadswell, H. E., and Perry, D. H. (1963). “Assessment of wood qualities for tree breeding," Silvae Genetica 12, 105-110. 
Ogbonnaya, C. I., Roy-Macauley, H., Nwalozie, M. C., Annerose, D. J. M. (1997). "Physical and histochemical properties of kenaf (Hibiscus cannabinus L.) grown under water deficit on a sandy soil," Ind. Crop Prod. 7, 9-18.

Panshin, A. J., and De Zeeuw, C. (1970). “Textbook of wood technology,” Vol. I. McGraw-Hill, New York.

Panshin, A. J., and De Zeeuw, C. (1980). "Textbook of wood technology," 4ed. New York, Mc Graw-Hill, 722 pp.

Papamichael, P., and Paraskevopoulou, A. H. (1982). "Study of the physical properties of Cypress wood (Cupressus sempervirens var. horizontalis)," Dasiki Erevna (Forest Research), Vol. 3(1).

Paraskevopoulou, A. H. (1991). "Variation of wood structure and properties of Cupressus sempervirens var. horizontalis in natural populations n Greece," IAWA Bull. 12(2), 195-206.

Simpson, W. T. (1991). “Dry kiln operator's manual,” USDA For. Ser. Agric. Handb. 188. Madison, WI: U.S. Department of Agriculture, Forest Service, Forest Products Laboratory, $174 \mathrm{p}$.

Tischler, K. (1981). "Physical and mechanical properties of Cypress timber," Division of Forestry, Scientific activities 1977-1980, Special publ. No. 200.

Tsehaye, A., Buchanan, A. H., and Walker, J. C. F. (1995b). "A comparison of density and stiffness for predicting wood quality," Or Density: The lazy man's guide to wood quality. Journal of the Institute of Wood Science 13(6), 539-543.

TS 2476, (1976). "Odunda fiziksel ve mekanik deneyler icin rutubet miktarı tayini," Türk Standartları Enstitüsü (TSE), Ankara.

Uzielli, E., and Berti, R. N. (1979). "Aspetti technologici dellegno di Cipresso (Cupressus sempervirens L.),” In: II Cipresso: Malattiee DiCesa (Grasso, V., and Raddi, P. eds.), 95-109. Seminario CEE AGRIMED, Firenze.

Walker, J. C. F., and Butterfield, B. G. (1996). "The importance of the microfibril angle for the processing industrie," New Zealand Forestry, Feb. 1996, 34-40.

Wang, E., Chen, T., Pang, S., Karalus, A. (2008). "Variation in anisotropic shrinkage of plantation-grown Pinus radiata wood," Maderas. Ciencia y Tecnología 10(3), 243249, Universidad del Bío-Bío, Concepción, Chile.

Ward, D. (1975). "The influence of tree spacing upon tracheid length and density in Sitka spruce (Picea sitchensis (Bong.) Carr),” M. Agr. Sc. Thesis, University College Dublin.

Yanchuk, A. D., Dancik, B. P., and Micko, MM. (1984). "Variation and heritability of wood density and fiber length of trembling aspen in Alberta," Canada. Silv. Genet. $33,11-16$.

Article submitted: December 5, 2010; Peer review completed: January 15, 2011; Revised version received and accepted: April 6, 2011; Published: April 8, 2011. 